\title{
Cranial Neuroimaging and Clinical Neuroanatomy
}

\author{
Eugen Boltshauser ${ }^{1}$ \\ ${ }^{1}$ Department of Pediatric Neurology, University Children's Hospital, \\ Zurich, Switzerland \\ Neuropediatrics 2019;50:209-210.
}

H. Lanfermann, P. Raab, HJ Kretschmann, W. Weinrich. Cranial Neuroimaging and Clinical Neuroanatomy. Atlas of MR Imaging and Computed Tomography. Fourth Edition. Stuttgart: Thieme Publishers; 2019 (513 pages, 930 illustrations). ISBN 978-3-13-672604-4. EUR ca 200.00 (e-book: eISBN 978-3-13-241817-2)

This book represents an authorized translation of the fourth German edition published in 2015, a completely revised and updated edition compared with the previous edition from 2004. The team of authors (two neuroradiologists, a neuroanatomist, and a neurologist) come from Hannover (Germany).

The volume is organized in five (major) parts. Part I (introduction) includes very important information about the exact orientation of the (sagittal, transverse, and coronal) planes as these may differ in various (imaging and anatomic) atlases. It is important to realize that the transverse (axial) planes of magnetic resonance imaging (MRI) and computed tomography (CT) often differ. Part II (atlas) provides 14 coronal sections, six sagittal sections, 14 transverse MR sections, 14 transverse $\mathrm{CT}$ sections, and 10 brainstem sections.

Anatomical atlas illustrations and MR and CT images were placed in the same size as large-format side-by-side. In the coronal sections (as example) two anatomical images at the same level are presented, one with brain structures and cranial nerves and the other with bony structures, muscles, and blood vessels named, while the corresponding neuroimage is given as separate T1-weighted and T2-weighted MRI. This allows perfect comparison and identification of the structures. The
Address for correspondence Prof. em. Eugen Boltshauser Department of Pediatric Neurology, University Children's Hospital, Steinwiesstrasse 75, 8032 Zürich, Switzerland (e-mail: eugen.boltshauser@bluewin.ch).

quality of the figures and the naming of the anatomic details is excellent. Part III (topography of the head and neck) covers in detail topography of the cranium, intracranial spaces (as cisterns and ventricular system), and contained structures (as cerebral arteries and their vascular territories, cerebral veins, and cranial nerves). Detailed anatomic figures, as well as anatomic images, with corresponding structures highlighted in colors, projected into schematic MR figures (i.e., vascular territories in three planes) are provided. A chapter on brain maturation is included, with detailed illustration of myelination including T1-weighted and T2-weighted images for the first 24 months. Part IV is mainly devoted to neurofunctional systems (100 pages). The systems (sensory, gustatory, ascending reticular system, vestibular, auditory, visual, olfactory, motor, cerebellar, speech areas, limbic system, autonomic nervous system, and neuronal networks) are described and illustrated in detail. The tracts/pathways are highlighted in schematic drawings and its locations in corresponding MRI schemes is evidently presented (i.e., the anatomic location of the visual system is shown in multiple MR sections in all three planes. In Part V, (appendix) the authors give account of the origin of the specimens used from the neuroanatomical collection, the images obtained from a few deceased individuals, as well those gained from living persons.

Neuroanatomy is the basis of imaging interpretation. This detailed atlas in large printed format is an excellent "tool" for this endeavor for anyone interested in this task. It was a personal pleasure thumbing through this beautiful aesthetic book. Access for the complimentary online version is possible. published online

April 2, 2019 (c) 2019 Georg Thieme Verlag KG Stuttgart · New York
DOI https://doi.org/ 10.1055/s-0039-1685215. ISSN 0174-304X. 